\title{
On the Intersection of Finitely Generated Subgroups of Free Groups
}

\author{
W.S. JASSIM \\ To A.H.M. Hoare
}

\begin{abstract}
Howson [4] proved that the intersection of two finitely generated subgroups $H$ and $K$ of ranks $m$ and $n$ respectively is finitely generated. He proved that the rank $N$ of $H \cap K$ is at most $2 m n-m-n+1$. H. Neumann [8,9] gave a better bound of $2 m n-2 n-2 m+3$. Burns [2] further improved the general upper bound to $N \leq 2 m n-3 m-2 n+4$ (for $m \leq n$ ).

Imrich [6] gave shorter proof of Neumann's result and also Nickolas [10] gave simple proof for Burn's result. Servatius [12] gave graphical proof for Burn's result.
\end{abstract}

Burns [1] showed that the stronger bound $N \leq m n-n-m+2$ holds if $H$ or $K$ is of finite index in $F$.

In this paper it is shown that stronger bound $N \leq m n-n-m+2$ always hold.

In section 1 we gave basic concepts about free groups, graphs and cayley graphs.

1991 Mathematics Subject Classification: 20E07

Servicio publicaciones Univ. Complutense. Madrid, 1996.

I would like to thank the referee for his helpful suggestions. 
In section 2 we showed the main theorem 2.7: "If $H$ and $K$ are finitely generated subgroups of a free group $F$ on generators $a, b$ of ranks $m, n$ respectively and if all vertices in $\Gamma^{*}(H)$ and $\Gamma^{*}(K)$ are of degree 2 and 3 only, then the rank $N$ of $H \cap K$ satisfies $N \leq m n-n-m+2$ ".

In order to prove the main Theorem 2.7, we followed and improved the techniques which were used by Nickolas [10] especially the concept of compatibelty of paths and branch points.

By this improvement, we could have an upper bound on the number of the compatible branch points in the core of $\Gamma^{*}(H) \tilde{x} \Gamma^{*}(k)$ which is the product of $\Gamma^{*}(H)$ and $\Gamma^{*}(k)$.

Therefore we started to know the least number of typing of compatible branch points of degree 3 only in $\Gamma^{*}(H)$ as shown in Lemma 2.3 .

In Lemma 2.4 we showed that if $\Gamma^{*}(H)$ has only two types of compatible branch points $X_{1}$ and $X_{2}$ then the number of branch points of type $X_{1}=$ the number of branch points of type $X_{2}$.

In Lemma 2.5 we showed that if $\Gamma^{*}(H)$ has more than two types of compatible branch points then the largest number of one type of compatible branch points is $n$, where \# $\mathrm{Br}\left(\Gamma^{*}(H)=2 n \geq 4\right.$ or $i<n$ for all $i=1, \ldots, r$, where $r$ is the number of typing of compatible branch point $X_{1}$.

Therefore by above Lemmas $2.3,3.4$ and 2.5 we could have an upper bound for the number of branch points in the core of $\Gamma^{*}(H) \tilde{\times} \Gamma^{*}(K)$ which is less than or equal to $\frac{\# B r\left(\Gamma^{*}(H)\right) \times \# B r\left(\Gamma^{*}(K)\right)}{2}$ as shown in Theorem 2.6.

\section{INTRODUCTION}

\subsection{Free groups}

A group $F$ is said to be free on a finite subset $X \subseteq F$, where $X=\left\{x_{1}, x_{2}, \ldots, x_{n}\right\}$ if for any group $B$ and any mapping $f: X \rightarrow G$ there is a unique homomorphism $\theta: F \rightarrow G$ such that $x \theta=x f$ for all $x \in X$.

The cardinality of $X$ is called the rank of $F$ and is denoted by $|X|$; and $X$ is called a set of generators of the free group $F$. If $X$ is finite then $F$ is called finitely generated free group. 
A word on $X$ is a finite sequence of elements in $X^{+} \cup X^{-}$where $X^{-}=\left\{x_{1}^{-1}, x_{2}^{-1}, \ldots, x_{n}^{-1}\right\} X=X^{+}=\{x: x \in X\}$. A word is denoted by $W$. A word $W=x_{i_{1}}^{\epsilon_{1}} x_{i_{2}}^{\epsilon_{2}} \ldots x_{i_{r}}^{\epsilon_{r}}$ when $1 \leq i \leq n, r \geq 0, \epsilon= \pm 1$, is called a reduced word or a finitely reduced word if $x_{i_{j}}^{\epsilon_{j}} \neq x_{i_{i+1}}^{\epsilon_{j+1}}$.

The set of all reduced words is denoted by $F_{x}$. The inverse of the word $W=x_{i_{1}}^{\epsilon_{1}} x_{i_{2}}^{\epsilon_{2}} \ldots x_{i_{r}}^{\epsilon_{r}}$ is the word $x_{i_{r}}^{-\epsilon_{r}} x_{i_{r-1}}^{-\epsilon_{r-1}} \ldots x_{i_{2}}^{-\epsilon_{2}} x_{i_{1}}^{-\epsilon_{1}}$ and is denoted by $W^{-1}$. The length of the word $W_{1}$ is the length $r$ of the finite sequence $x_{i_{1}}^{\epsilon_{1}} x_{i_{2}}^{\epsilon_{2}} \ldots x_{i_{r}}^{\epsilon_{r}}, \epsilon= \pm 1$ and is denoted by $L(W)$. The empty word has length zero, i.e. $L(1)=0,1$ is the identity element of $F$. It is clear that $L(W)=L\left(W^{-1}\right)$.

The words $W_{1}$ and $W_{2}$ on $X$ are called equivalent (denoted by $W_{1} \sim W_{2}$ ) if the following operations applied a finite number of times change $W_{1}$ into $W_{2}$ or $W_{2}$ into $W_{1}:$ (1) Insertion of one of the words $V V^{-1}$ between any two consecutive symbols of $W$, or before $W$ or after $W$. (2) Deletion of one of the words $V V^{-1}$ if it forms a block of consecutive symbols in $W$. Nielson - Schreier [11] showed that if $F$ is a free group of rank $n$ and $H$ is a subgroup of $F$, then $H$ is free. If $|F: H|=g$ is finite, then the rank of $H$ is equal to $g(n-1)+1$.

\subsection{Graphs}

A graph $\Gamma$ is a collection of two sets $V$ ( $V$ is not empty set) and $E$ called the set of vertices and edges respectively of the graph $\Gamma$, together with two functions $i: E \rightarrow V, t: E \rightarrow V$ (we say that edge $e$ joins the vertex $i(e)$ to $t(e)$. The vertex $i(e)$ is called the initial vertex of $e$ and $t(e)$ is called the terminal vertex of $e$ ). Moreover for each $e$ in $E$ there is an element $\bar{e} \neq e$ in $E$, called the inverse of $e$, such that $i(\vec{e})=t(e)$, $t(\bar{e})=i(e)$ and $\overline{\bar{e}}=e$.

A subgraph $\Delta$ of a graph $\Gamma$ is a graph with $V(\Delta) \subseteq V(\Gamma), E(\Delta) \subseteq$ $E(\Gamma)$. If $e \in E(\Delta)$, then $i_{\Delta}(e), t_{\Delta}(e)$ and $\bar{e}$ have the same meaning in $\Gamma$ as they do in $\Delta$. If $\Delta \neq \Gamma$ then we call $\Delta$ a proper subgraph.

A path $P$ in a graph $\Gamma$ is a finite sequence $e_{1}, e_{2}, \ldots, e_{n}$, where $e_{i} \in E(\Gamma), 1 \leq i \leq n-1, t\left(e_{i}\right)=i\left(e_{i+1}\right)$.

The initial vertex of $P$ is the initial vertex of $e_{1}$ and the terminal vertex of $P$ is the terminal vertex of $e_{n}$. The path $P$ is called reduced path if $e_{i} \neq \bar{e}_{i+1}$, for $\leq i \leq n-1$ where $P=e_{1} e_{2} \ldots e_{n}, e_{i} \in E(\Gamma)$ and 
closed if $i(P)=t(p)$. If $P$ is reduced and closed, then $P$ is called a circuit or cycle.

The length of a path $P$ is the number of edges in the path $P$. If $P_{1}$ and $P_{2}$ are paths in a graph $\Gamma$ and the terminal of $P_{1}$ equals the initial vertex of $P_{2}$, they may be concatenated to form a path $P_{1} P_{2}$ with $L\left(P_{1} P_{2}\right)=L\left(P_{1}\right)+L\left(P_{2}\right)$ such that $i\left(P_{1} P_{2}\right)=i\left(P_{1}\right), t\left(P_{1} P_{2}\right)=t\left(P_{2}\right)$, $t\left(P_{1}\right)=i\left(P_{2}\right)$.

A trivial successions of edges is a path of the form $e \bar{e}, \bar{e} e$, where $e \in E, \bar{e} \in E$.

If a path $P$ contains a trivial succession of edges then by collapsing the trivial successions of edges we get a new path $P^{\prime}$. This operation is called an elementary reduction and is denoted by $P \downarrow P^{\prime}$.

Two paths $P$ and $P^{\prime}$ are called equivalent, denoted by $P \sim P^{\prime}$, if there is a finite sequence of paths $P=P_{1}, P_{2}, \ldots, P_{k}=P^{\prime}$ such that either $P_{j} \downarrow P_{j+1}$ or $P_{j+1} \downarrow P_{j}$ for $i \leq j \leq k-1$. Therefore as in [3], (a) Each path $P$ is equivalent to a unique reduced path. (b) the operation of composition of paths is compatible with equivalence. That is, $P \sim P^{\prime}$, $S \sim S^{\prime}$ implies $P S \sim P^{\prime} S^{\prime}$, if the compositions are defined.

A graph $\Gamma$ is connected if $v, u \in V(\Gamma)$ implies there exists a path in $\Gamma$ joining $v$ to $u$. A component of $\Gamma$ is a maximal connected subgraph of $\Gamma$.

A tree is a connected non-empty graph without reduced circuits.

If $\Delta$ is a subgraph of a connected graph $\Gamma$, then $\Delta$ is called spanning if every pair of vertices of $\Gamma$ is joined by at least one path in $\Delta$ and a spanning tree if $\Delta$ is tree and spanning.

A morphism of graphs is a function $f: \Gamma \rightarrow \pi$ such that $f$ takes each edge to an edge or a vertex and each vertex to a vertex with the following property $f(\bar{e})=\overline{f(e)}$, where $e \in \Gamma$ and $i(f(e))=f(i(e))$ when $i(v)=v$ for $v \in V$.

Two graphs $\Gamma$ and $\pi$ are called isomorphic is there exists a one-one mapping $f$ of the vertices and edges of $\Gamma$ onto the vertices and edges respectively of $\pi$, which preserves the relation "is the initial vertex of", "is the terminal vertex of" and "is the inverse of". 


\subsection{Cayley graphs}

The graph $\Gamma(F, X)$ is called the cayley graph of the group $F$ with respect to $X \subseteq F$. It has vertex set $F$ and set of edges $F \times X$ (i.e. $(w, x) \in E(\Gamma(F, X))$ with $i(w, x)$ is the initial vertex of $(w, x)$ and $t(w, x)$ the termanal vertex of $(w, x)$ for every edge $(w, x) \in E(\Gamma(F, X))$, the inverse edge of $(w, x)$ is $\left(w x, x^{-1}\right) \in E(\Gamma(F, X))$.

The quotient graph [5] or cayley coset graph $\Gamma(F, X) / H$ for a subgroup $H$ of $F$ has set of vertices $\{H w: w \in F, H \leq F\}$ and set of edges $\{(H w, x): w \in F, x \in X\}$ such that an edge $(H w, x) \in$ $\Gamma(F, X) / H$ takes the vertex $H w$ to $H w x$. It is also denoted by $\Gamma(H)$. The core of a coset graph $\Gamma(H)$ is the smallest subgraph containing all cycles. It is denoted by $\Gamma^{*}(H)$, for example, if $F$ is a free group on generators $a, b$, then
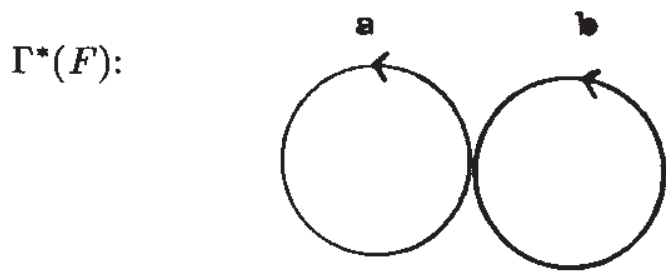

The number of cycles in $\Gamma^{*}(H)$ is called the cyclomatic number. The cyclomatic number of $\Gamma^{*}(H)$ is the minimal number of edges that we can delete to make a tree.

The rank of the finitely generated subgroup $H$ is the cyclomatic number of $\Gamma^{*}(H)$. The vertex $V$ is called a branch point if $d(V) \geq 3$ where $V \in V\left(\Gamma^{*}(H)\right)$ and $d(V)$ is the degree of the vertex $V$.

\section{MAIN THEOREM}

By direct calculations we can prove the following proposition.

Proposition 2.1. If $\Gamma^{*}(H)$ is a core graph of finitely generated subgroup $H$ of rank $m$ and if all vertices of $\Gamma^{*}(H)$ are of degree 2 and 3 only. Then $m=1+\frac{\# B r\left(\Gamma^{*}(H)\right)}{2}$, where \#Br( $\Gamma^{*}(H)$ is the number of branch points in $\Gamma^{*}(H)$.

Two branch points are called neighbours if they are connected by a (reduced) path which does not contain any branch point. 
Now free group $F$ generated by $a, b$ and $\Gamma^{*}(H)$ has vertices of degree 2,3 and 4 as in [12]. We can reduce the degree of vertices in $\Gamma^{*}(H)$ into vertices of degree 2 and 3 only (by isomorphically embedding $F$ into a free group $Q$ on $\{u, v\}$ via the map $\theta: F \rightarrow Q$ with $\theta(a)=u v^{-1}$ and $\theta(b)=v^{2}$ and taking the graph into new set of labels $\{u, v\}$.

The product of core graphs $\Gamma^{*}(H)$ and $\Gamma^{*}(k)$ (where $H$ and $K$ are finitely generated subgroups of the free group $F\{a, b\})$ is the graph $\Gamma^{*}(H) \tilde{\times} \Gamma^{*}(H)$ with set of vertices $V_{1} \times V_{2}=\left\{(v, u) ; v \in V_{1}\right.$ and $\left.u \in V_{2}\right\}$ and edges $\left\{((u, v), y) ;(v, y) \in E\left(\Gamma^{*}(H)\right)\right.$ and $(u, y) \in E\left(\Gamma^{*}\left(K^{*}\right)\right)$ and $i \in X\}$. Nickolas [10] showed the following:

Proposition 2.2. Let $\Gamma^{*}(H), \Gamma^{*}(K)$ and $\Gamma^{*}(H \cap K)$ be core graphs of $\Gamma(H), \Gamma(K)$ and $\Gamma(H \cap K)$ respectively. If $\Gamma^{*}(H) \tilde{\times} \Gamma^{*}(K)$ is the product of $\Gamma^{*}(H)$ and $\Gamma^{*}(K)$ defined above, then $\Gamma *(H \cap K)$ may be identified with core of a connected component of the graph $\Gamma^{*}(H) \tilde{\times} \Gamma^{*}(K)$. N.B. In the rest of the paper we will write core of $\Gamma^{*}(H) \widetilde{\times} \Gamma^{*}(K)$ to mean core of a connected component of the graph $\Gamma^{*}(H) \tilde{\times} \Gamma^{*}(K)$.

In $\Gamma^{*}(H)$ there are four possibilities for the branch points in the core of $\Gamma(H)$ as follows:


These branch points are called $a$-sources, $a$-sinks, $b$-sources and $b$ sinks respectively.

For any two paths $P_{i j}$ and $P_{r s}$ in $\Gamma^{*}(H) \cup \Gamma^{*}(K)$ (may $K=H$ ), we say $P_{i j}$ and $P_{r s}$ are compatible if $P_{i j}=P_{r s}$ and $P_{i j}$ or $P_{r s}$ or both of them may pass through several branch points such that $P_{i j}$ starting at a branch point $i$ and ending at a branch point $j$ and $P_{r s}$ starting at a branch point $r$ and ending at a branch point $s$ also $(i, r)$ and $(j, s)$ are neighbouring branch points in $\Gamma^{*}(H) \tilde{\times} \Gamma^{*}(K)$.

Therefore branch points $i, r$ in $\Gamma^{*}(H) \cup \Gamma^{*}(K)$ (may $K=H$ ) are called compatible if all paths $P_{i j}$ and $P_{r s}$ are compatible in $\Gamma^{*}(H) \cup$ 
$\Gamma^{*}(K)$ and $(i, r)$ and $\left(j_{t}, s_{t}\right)$ are neighbouring branch points in $\Gamma^{*}(H) \tilde{x}$ $\Gamma^{*}(K)$ for each $t=1,2,3$. (Incompatible will mean not compatible). We can write $P_{i j_{t}}=P_{t}=P_{r s_{t}}$ for each $t$. If $u_{1}$ and $u_{2}$ are compatible branch points in $\Gamma^{*}(H) \cup \Gamma^{*}(K)$ then $P_{t}$ are compatible for each $t$. Also if $\mathcal{U}_{2}$ and $\mathcal{U}_{3}$ are compatible branch points in $\Gamma^{*}(H) \cup \Gamma^{*}(K)$ then $q_{u_{2} k_{t}}=q_{t}=q_{u_{3} v_{t}}$ are compatible for each $t=1,2,3$.

Therefore $\left.L\left(P_{t}\right) \leq L\left(q_{t}\right)\right)$, where $L\left(P_{t}\right)$ and $L\left(q_{k}\right)$ are the length of the paths $P_{t}$ and $q_{t}$ respectively, $t=1,2,3$.

If $\mathcal{U}_{1}$ and $\mathcal{U}_{2}$ are compatible then $g_{u_{1} i_{i}}=g_{t}=g_{u_{9} h_{t}}$ are compatible for each $t=1,2,3$.

Therefore either $P_{t}=q_{t}=g_{t}$ or $g_{t}=q_{t}=P_{t} h_{t}$, where $t\left(P_{t}\right)=i\left(h_{t}\right)$ as in examples 1 and 3 .

The following example show us that the compatibility of branch points is not transitive in general.

We see that 1 and 2 are compatible. Also 2 and 6 are compatible but 1 and 6 are incompatible.

Definition. A consistent graph is a directed $X$-labelled graph (where $\left.X=\left\{x_{1}, x_{2}, \ldots, x_{n}\right\}\right)$ with no reduced paths labelled $x_{i}, x_{i}^{-1}$ or $x_{i}^{-1}$, $x_{i}, x_{i} \in X \cup X^{-1}, 1 \leq i \leq n$.

If $X=\{a, b\}$, then no reduced paths labelled $a a^{-1}, b b^{-1}, a^{-1} a$ and $b^{-1} b$ ever occur in a consistent graph.

Lemma 2.3. Let $\Gamma^{*}(H)$ be the core graph of the finitely generated subgroup $H$ of the free group $F$ on generators $a, b$. Then $\Gamma^{*}(H)$ has at least two types of compatible branch points, where all branch points are of degree 3 only.

Proof. Suppose $\Gamma^{*}(H)$ has only one type of compatible branch points. Then all branch points are of one type say $b$-cources.

Thus all possibilities for paths joining neighbouring branch points are:

$A=e_{1} \ldots e_{n}$ where $e_{1}$ is an edge labelled $b$ and $e_{n}$ labelled $a^{-1}$,

$B=e_{1} \ldots e_{m}$ where $e_{1}$ is an edge labelled $b$ and $e_{m}$ labelled $a$,

$C=e_{1} \ldots e_{k}$ where $e_{1}$ is an edge labelled $b$ and $e_{k}$ labelled $b^{-1}$ 
$D=e_{1} \ldots e_{t}$ where $e_{1}$ is an edge labelled $a$ and $e_{t}$ labelled $a$

$E=e_{1} \ldots e_{r}$ where $e_{1}$ is an edge labelled $a$ and $e_{r}$ labelled $a^{-1}$

$G=e_{1} \ldots e_{s}$ where $e_{1}$ is an edge labelled $a^{-1}$ and $e_{s}$ labelled $a$
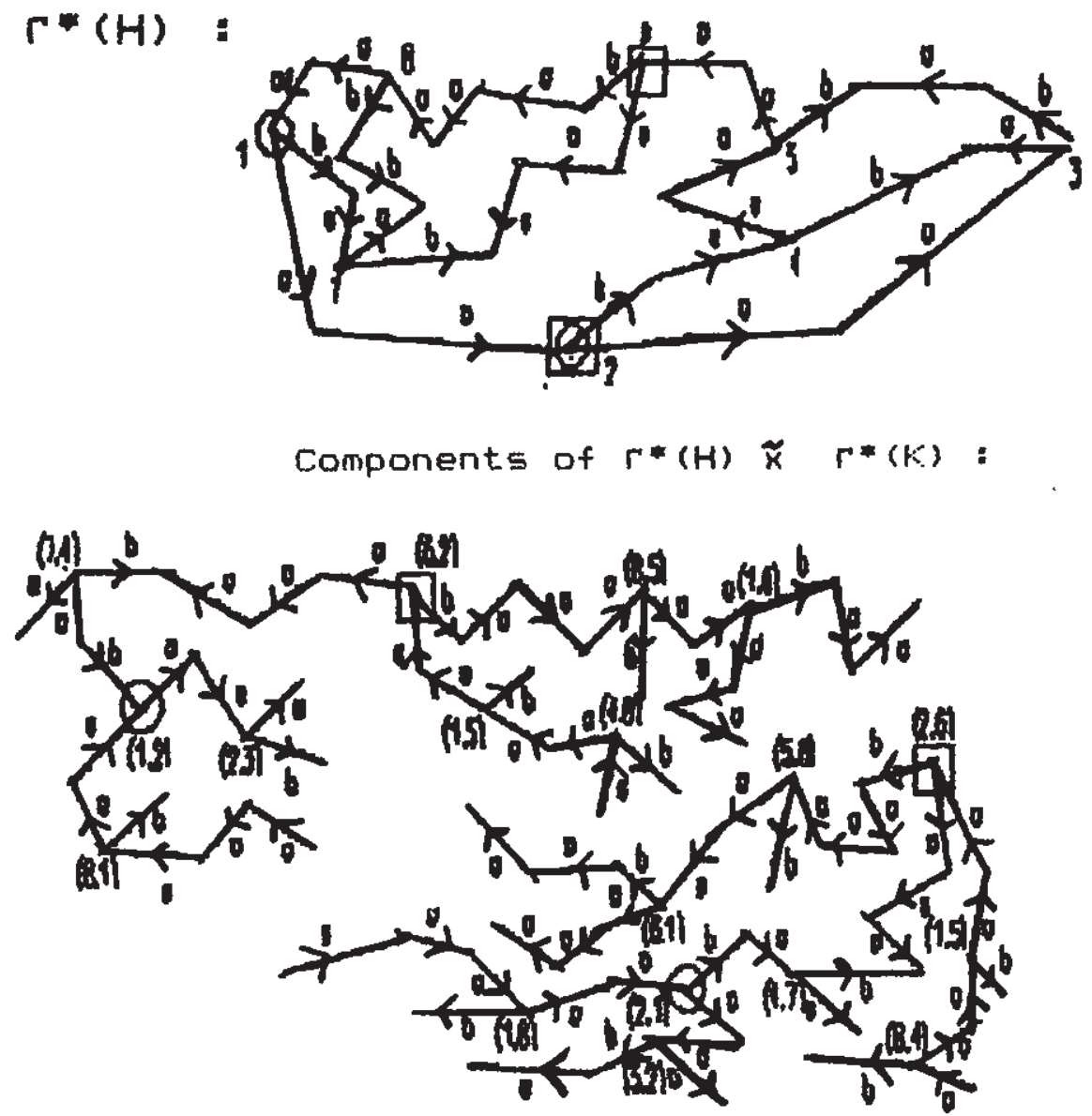

Since there are exactly three reduced paths $P_{1}, P_{2}$ and $P_{3}$ begining at each compatible branch point.

Let $P_{1}$ be a path starting with an edge labelled $b, P_{2}$ be a path starting with an edge labelled $a$ and $P_{3}$ be a path starting with an edge labelled $a^{-1}$.

Therefore all $P_{1}$ are compatible, all $P_{2}$ are compatible and all $P_{3}$ are compatible. 
Since $\Gamma^{*}(H)$ is a consistent graph so no path of type $C, E$ or $G$ contains a subpath labelled $a^{-1} a, a a^{-1}, b b^{-1}$ or $b^{-1} b$.

Thus each path $C, E$ and $G$ contains at least one subpath labelled ba. Then $\Gamma^{*}(H)$ does not contain paths of types $C, E$ or $G$ since $C \neq$ $C^{-1}, E \neq E^{-1}$ and $G \neq G^{-1}$ otherwise we will have more than one type of compatible branch points.

Therefore $\Gamma^{*}(H)$ has only paths of types $A, B$ and $D$.

Then $A=B V$ or $B=A Y$

Suppose $A=B V$. Therefore $A_{j}=B_{i} V_{j}$ for each $i, j$.

Therefore $P_{1}$ ending with an edge labelled $a^{-1}, a$ or $b^{-1} . V_{j}=b \ldots a^{-1}$ or $V_{j}=b^{-1} \ldots a^{-1}$ or $V_{j}=a \ldots a^{-1}$.

I) Suppose $V_{j}=b \ldots a^{-1}$

Therefore there exists $P_{1}=u_{0} B_{1} u_{1} A_{j} u_{2} \ldots u_{n}$ and

$$
P_{1}=u_{1} A_{j} u_{2} \ldots u_{m}
$$

Thus $A_{j}=B_{i} V_{j}$ and $B_{i}=V_{j} Z_{i}$, when $Z_{i}=b \ldots a, Z_{i}=b^{-1} \ldots a$ or $Z_{i}=a^{-1} \ldots a$.

Therefore

$$
\begin{aligned}
& P_{1}=u_{0} V_{j} Z_{i} u_{1} V_{j} Z_{i} V_{j} u_{2} \ldots u_{n} \\
& P_{1}=u_{1} V_{j} Z_{i} V_{j} u_{2} Z_{i} V_{j} \ldots u_{3} \ldots u_{m} \text { and } \\
& P_{1}=u_{2} Z_{i} V_{j} \ldots u_{3} \ldots u_{k}
\end{aligned}
$$

Now suppose $Z_{i}=b \ldots a$.

Since $L\left(Z_{i} V_{j}\right)=L\left(V_{j} Z_{i}\right)$ and $V_{j} Z_{i}$ and $Z_{i} V_{j}$ have different ending of edges so $u_{1}$ and $u_{2}$ are incompatible.

If $Z_{i}=b^{-1} \ldots a$ then $u_{2}$ is a branch point of type $b$-sink a contradiction.

Therefore $Z_{i}=a^{-1} \ldots a$

Thus all $P_{1}$ are compatible

Let $D_{r}^{-1}=Z_{i} V_{j}$ for some $r$, where $D_{r}$ is a path of type $D$. Since $D_{r}^{-1}$ and $B_{i}^{-1}$ starting with an edge labelled $a^{-1}$ so there exists at least two branch points $u_{1}$ and $u_{2}$ such that $D_{\tau}^{-1}$ beginning at $u_{2}$ and $B_{i}^{-1}$ begining at $u_{1}$. 
Since $D_{r}^{-1}=Z_{i} V_{j}$ and $B_{i}^{-1}=Z_{i}^{-1} V_{j}^{-1}$ and

$Z_{i}=a^{-1} \ldots b \ldots a \neq a^{-1} \ldots b^{-1} \ldots a=Z_{i}^{-1}$ so

$u_{1}$ and $u_{2}$ are incompatible.

II) Suppose $V_{j}=a \ldots a^{-1}$

Since $A_{j}=B_{j} V_{j}$ so $B_{i} \neq V_{j} Z_{i}$.

Since $P_{1}=u_{0} B_{i} u_{1} V_{j} u_{2} \ldots u_{n}$ and $P_{1}=u_{1} B_{i} V_{j} u_{2} \ldots u_{m}$ and $B_{i}$ and $V_{j}$ have different starting of edges so $u_{0}$ and $u_{1}$ are incompatible.

III) Suppose $V_{j}=b^{-1} \ldots a^{-1}$

Since $A_{j}=B_{i} V_{j}$ so $B_{i} \neq V_{j} Z_{i}$.

Since $P_{1}=u_{0} B_{i} u_{1} B_{i} V_{j} u_{2} \ldots u_{n}, P_{1}=u_{1} B_{i} V_{j} u_{2} \ldots u_{m}$ so $u_{0}$ and $u_{1}$ are incompatible.

Similarly we have the same result if $B_{i}=A_{j} Y_{i}$.

Also all other cases will give the same result.

If $\Gamma^{*}(H)$ has more than or type of branch points then $\Gamma^{*}(H)$ has at least two types of compatible branch points. Thus $\Gamma^{*}(H)$ has at least two types of compatible branch points.

Lemma 2.4. Let $\Gamma^{*}(H)$ be the core graph of finitely generated subgroup $H$ of the free group $F$ on generators $a, b$. If $\Gamma^{*}(H)$ has only two types of compatible branch points $X_{1}$ and $X_{2}$ then $x_{1}=x_{2}$ where $x_{1}$ and $x_{2}$ are the number of the compatible branch points of types $X_{1}$ and $X_{2}$ respectively and $\Gamma^{*}(H)$ has $2 n=x_{1}+x_{2}$ branch points.

\section{Proof.}

If $n=2$

Then by Lemma 2.3, we have two types of compatible branch points. Thus $x_{1}=x_{2}$.

Suppose $x_{1}=x_{2}$ for $m=2 k<2 n$.

Now we prove $x_{1}=x_{2}$ for $m+2=2 k+2$.

Suppose $u_{k+1}$ and $u_{2 k+2}$ are compatible branch points and of types $b$ sources.

I) If all branch points are of one type $b$ - sources say, as in proof of Lemma $2.3 \Gamma^{*}(H)$ has paths of type $A$ and $G$ only or $B$ and $E$ only or 
$C$ and $D$ only or $A, B$ and $D$ only otherwise will have more than two types of compatible branch points.

i) If $\Gamma^{*}(H)$ has paths of type $A$ and $G$ only.

There is a path of type $A$ or $G$ joining two neighbouring branch points $u_{j}$ and $u_{r}$ in $\Gamma^{*}(H), j, r \leq m$.

Let a path of type $A$ joining the branch points $u_{j}$ and $u_{2 k+1}$ then the path of type $A$ joins $u_{2 k+2}$ and $u_{r}$.

Then $u_{2 k+1}$ and $u_{2 k+2}$ should join by paths of types $E$ and $G$ a contradiction. If the path $G$ joins $u_{j}$ and $u_{2 k+1}$ then $G$ joins $u_{2 k+2}$ and $u_{r}$. Therefore the path $P_{3}$ beginning at $u_{2 k+1}$ and starting with a subpath of type $G^{-1}$, also $P_{3}$ beginning at $u_{2 k+2}$ and starting with a subpath of type $G$ since $G \neq G^{-1}$ so $u_{2 k+1}$ and $u_{2 k+2}$ are incompatible.

Similarly

We will have a contradiction if $\Gamma^{*}(H)$ contains paths of types $B$ and $E$ or $C$ and $D$ only.

ii) If $\Gamma^{*}(H)$ has paths of types $A, B$ and $D$ only. Then there is a path of type $A$ or $B$ or $D$ joins two neighbouring branch points $u_{j}$ and $u_{r}, j, r \leq m$.

Let a path of type $A_{j}$ joins $u_{j}$ and $u_{2 k+1}$ then the path $A_{j}$ joins $u_{2 k+2}$ and $u_{r}$ and suppose $A_{j}=B_{i} V_{i}$. Therefore the path of type $B_{i}$ should joins $u_{2 k+2}$ and $u_{2 k+1}$ otherwise we will have a contradiction. Therefore as in proof of Lemma 2.3 we have $A_{j}=B_{i} V_{j}$ and $B_{i}=$ $V_{i} Z_{i}, Z_{i}=a^{-1} \ldots a, V_{j}=b \ldots a^{-1}$.

Thus $P_{1}=u_{j} V_{j} Z_{i} V_{j} u_{2 k+1} V_{j} Z_{i} u_{2 k+2} V_{j} Z_{i} V_{j} u_{r} \ldots u_{n}$,

$P_{1}=u_{2 k+1} V_{j} Z_{i} u_{2 k+2} V_{j} Z_{i} V_{j} u_{\tau} \ldots u_{m}$,

$P_{1}=u_{2 k+2} V_{j} Z_{i} V_{j} u_{r} Z_{i} V_{j} \ldots u_{k}$

$P_{3}=u_{2 k+1} D_{r}^{-1} u_{2 k+2} \ldots u_{g}$

$P_{3}=u_{2 k+2} B_{i}^{-1} u_{2 k+1} \ldots u_{h}$

since $D_{r}^{-1}=a^{-1} \ldots a^{-1}, B_{i}^{-1}=Z_{i}^{-1} V_{j}^{-1}=a^{-1} \ldots a a \ldots b^{-1}$ so $B_{i}^{-1}=D_{r}^{-1} C_{i}$ or $D_{r}^{-1}=B_{i}^{-1} E_{r}$.

(1) Suppose $D_{r}^{-1}=B_{i}^{-1} E_{r}$ and $E_{r}=a^{-1} \ldots a^{-1}$

$P_{3}=u_{2 k+1} B_{i}^{-1} E_{r} u_{2 k+2} B_{i}^{-1} u_{2 k+1} E_{r} B_{i}^{-1} \ldots u_{g}$ 
Since $L\left(B_{i}^{-1} E_{r}\right)=L\left(E_{r} B_{i}^{-1}\right)$ and have different ending so $u_{2 k+1}$ and $u_{2 k+1}$ are incompatible.

Thus $u_{2 k+1}$ and $u_{2 k+2}$ are incompatible.

(2) Suppose $D_{r}^{-1}=B_{i}^{-1} E_{r}$ and $E_{r}=a \ldots a^{-1}$

Thus $P_{3}=u_{2 k+1} B_{i}^{-1} E_{r} u_{2 k+2} B_{i}^{-1} u_{2 k+1} E_{r} B_{i}^{-1} \ldots u_{g}$

Since $A_{j}^{-1}$ starting with an edge labelled $a$ and $E_{r} B_{i}^{-1}$ starting with an edge labelled $a$ so $A_{j}^{-1}=E_{r} B_{i}^{-1}$ and then $E_{r}=V_{j}^{-1}$ a contradiction since $E_{r}$ and $V_{j}^{-1}$ have different ending of edges.

Thus $u_{2 k+1}$ and $u_{2 k+2}$ are incompatible.

Similarly we have some result if $V_{j}=a \ldots a^{-1}$ and also if $B_{i}^{-1}=$ $D_{r}^{-1} F$.

Thus $x_{1}=x_{2}$.

II) Suppose $\Gamma^{*}(H)$ has two different types of branch points $b$-sources and $b$-sinks say.

It $n=2$ then $x_{1}=x_{2}$.

Suppose $x_{1}=x_{2}$ for $m=2 k<2 n+2$.

Thus all possibilities for paths joining neighbouring branch points are:

$A=e_{1} \ldots e_{n}$ where $e_{1}$ is an edge labelled $b$ and $e_{n}$ labelled $a^{-1}$

$B=e_{1} \ldots e_{m}$ where $e_{1}$ is an edge labelled $b$ and $e_{m}$ labelled $a$

$D=e_{1} \ldots e_{t}$ where $e_{1}$ is an edge labelled $a$ and $e_{t}$ labelled $a$

$G=e_{1} \ldots e_{i}$ where $e_{1}$ is an edge labelled $b^{-1}$ and $e_{i}$ labelled $a$ and

$K=e_{1} \ldots e_{j}$ where $e_{1}$ is an edge labelled $b^{-1}$ and $e_{j}$ labelled $a^{-1}$.

All other possibilities of paths will give more than two types of compatible branch points.

Since all branch points of type $b$-sources are compatible so there are exactly three paths $P_{1}, P_{2}$ and $P_{3}$ defined as before. Since all branch points of type $b$-sinks are compatible so there are exactly three paths $q_{1}, q_{2}$ and $q_{3}$ beginning at $b$-sinks.

Let $q_{1}$ be a path starting with an edge labelled $b^{-1}$

$q_{2}$ be a path starting with an edge labelled $a$ and

$q_{3}$ be a path starting with an edge labelled $a^{-1}$

Let $u_{j} \& u_{r}$ be two neighbouring branch points in $\Gamma^{*}(H), j, r \leq m$. 
i) If $u_{j}$ and $u_{r}$ are joined by paths of types $A, B$ or $D$ then as in proof of Lemma 2.3 we have $u_{2 k+1}$ and $u_{2 k+2}$ are incompatible.

ii) If $u_{j}$ and $u_{r}$ are joined by a path of type $G$ then $u_{j}$ and $u_{2 k+1}$ are joined by $G$ and then $u_{2 k+2}$ and $u_{r}$ are joined by $G$ a contradiction. Thus $u_{2 k+1}$ and $u_{2 k+2}$ are incompatible.

Similarly we have a contradiction if $u_{j}$ and $u_{2 k+1}$ are joined by a path $k$.

Thus $x_{1}=x_{2}$

Similarly we have $x_{1}=x_{2}$ if $u_{2 k+1}$ and $u_{2 k+2}$ are compatible of type $b$ - sinks.

Lemma 2.5. Let $\Gamma^{*}(H)$ be the core graph of the finitely generated subgroup $H$ of the free group $F$ on generators $a, b$. If $\Gamma^{*}(H)$ has more than two types of compatible branch points then the largest number of one type of compatible branch points is $n$, where \#Br $\left(\Gamma^{*}(H)=2 n \geq 4\right.$ or $x_{i}<n$ for all $i=1,2 \ldots r$, where $r$ is the number of typing of compatible branch points $X_{i}$.

Proof. Suppose $\Gamma^{*}(H)$ has only three types of compatible branch points $X_{1}, X_{2}$ and $X_{3}$.

Let $x_{i}$ be the number of compatible branch points of type $X_{i}, i=1,2,3$. Let $x_{1}$ be the largest number of compatible branch points.

If $2 n=4$ then $x_{1}=n$, otherwise we have $x_{i}<n$ for all $i, i=1,2,3,4$.

Now suppose $x_{1}=k$ for $m=2 k<2 n$.

Similarly as in proof of Lemma 2.4 then $x_{1}=k+1$ for $m=2 k+2$, therefore $x_{1}=n$.

Thus by induction on the number of typing of compatible branch points we have $x_{1}=n$ and $x_{2}+x_{3}+\ldots+x_{\tau}=n$ or $x_{i}<n$ for all $i=1, \ldots r$.

Theorem 2.6. Let $\Gamma^{*}(H)$ and $\Gamma^{*}(K)$ be the core graphs of the finitely generated subgroups $H$ and $K$ of the free group $F$ on generators $a, b$.

If $\Gamma^{*}(H) \cup \Gamma^{*}(K)$ has at least two types of compatible branch points then 


$$
\# B r\left(\operatorname{core} \Gamma^{*}(H) \tilde{x} \Gamma^{*}(K)\right) \leq \frac{\# B r\left(\Gamma^{*}(H)\right) \times \# B r\left(\Gamma^{*}(K)\right)}{2}
$$

Proof. Since the product of incompatible branch points is a vertex of degree at most 2 or a branch point is not in core of $\Gamma^{*}(H) \tilde{x} \Gamma^{*}(K)$ so by Lemmas 2.3 and 2.4

$$
\# B r\left(\operatorname{core} \Gamma^{*}(H) \tilde{x} \Gamma^{*}(K)\right) \leq 2 x_{1} y_{1}=\frac{\# B r\left(\Gamma^{*}(H)\right) \times \# B r\left(\Gamma^{*}(K)\right)}{2}
$$

or by Lemma 2.5

$$
\# B r\left(\operatorname{core} \Gamma^{*}(H) \tilde{\times} \Gamma^{*}(K)\right)<\frac{\# B r\left(\Gamma^{*}(H)\right) \times \# B r\left(\Gamma^{*}(K)\right.}{2}
$$

where $x_{i}$ and $y_{i}$ are the number of compatible branch points of types $X_{i}$ and $Y_{i}$ in $\Gamma^{*}(H)$ and $\Gamma^{*}(K)$ respectively, \# $X_{i} \leq \# Y_{i}$ and $X_{i}$ and $Y_{i}$ are compatible.

Theorem 2.7. If $H$ and $K$ are finitely generated subgroups of a free group $F$ on generators $a, b$ of ranks $m, n$ respectively and if all vertices in $\Gamma^{*}(H)$ and $\Gamma^{*}(K)$ are of degree 2 and 3 only, then the rank $N$ of $H \cap K$ satisfies $N \leq m n-m-n+2$.

Proof. By Theorem 2.6 and Propositions 2.1 and 2.2 the result follows.

Corollary 2.8 [1] If $H$ and $K$ are finitely generated subgroups of a free group $F$ on generators $a, b$ of rank $m, n$ respectively. If $H$ or $K$ has $a$ finite index in $F$ then the rank $N$ of $H \cap K$ satisfies $N \leq m n-n-m+2$.

Proof. If $H$ say, has a finite index in $F$, then $\Gamma^{*}(H)=\Gamma(H)$ and all vertices in $\Gamma^{*}(H)$ are of degree 4 only. 
Therefore as in [12], we can reduce the degree of vertices in $\Gamma^{*}(H)$ into vertices of degree 3 only. Thus we have a new consistent graph $\pi^{*}$ which has branch points of degree 3 only.

By Theorem 2.7 the result follows.

Example 1:
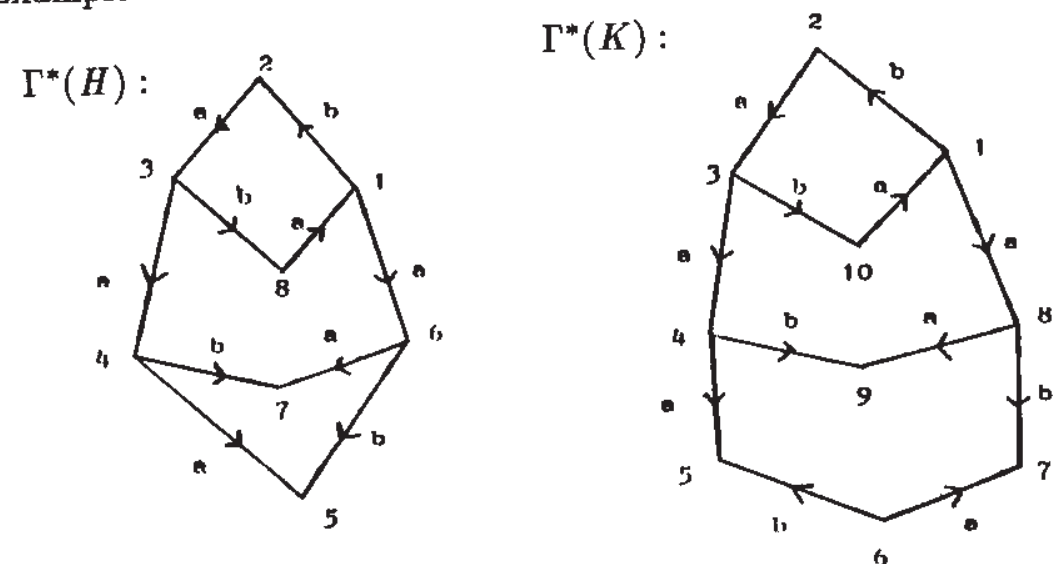

Core of $\Gamma^{*}(H) \tilde{\times} \Gamma^{*}(K)$ :

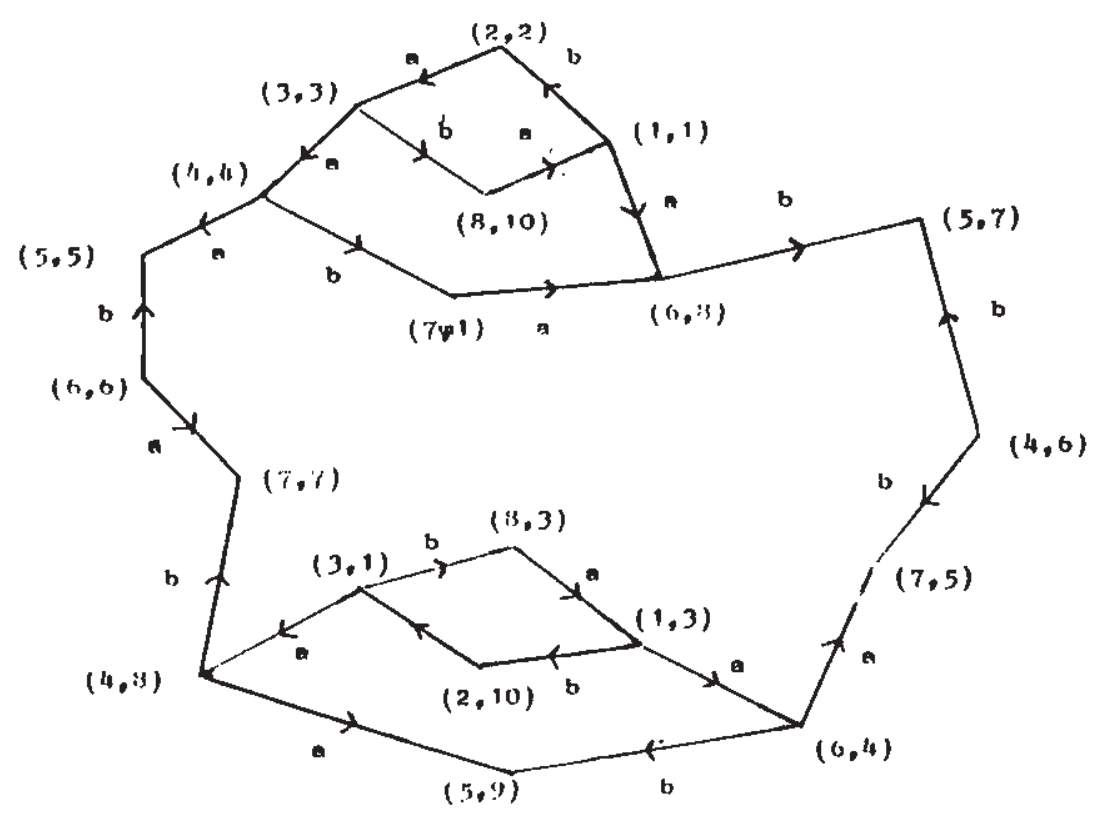


Example 2:

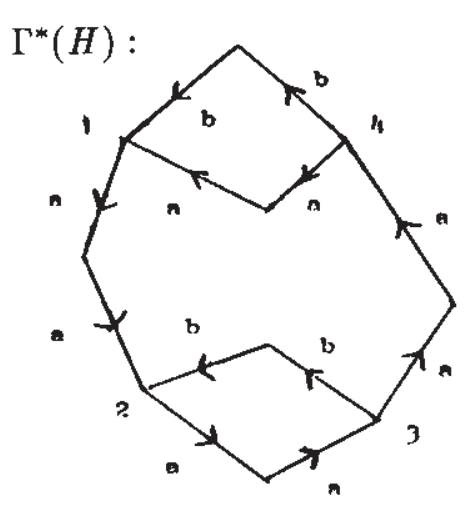

Core of $\Gamma^{*}(H) \tilde{x} \Gamma^{*}(K)$ :
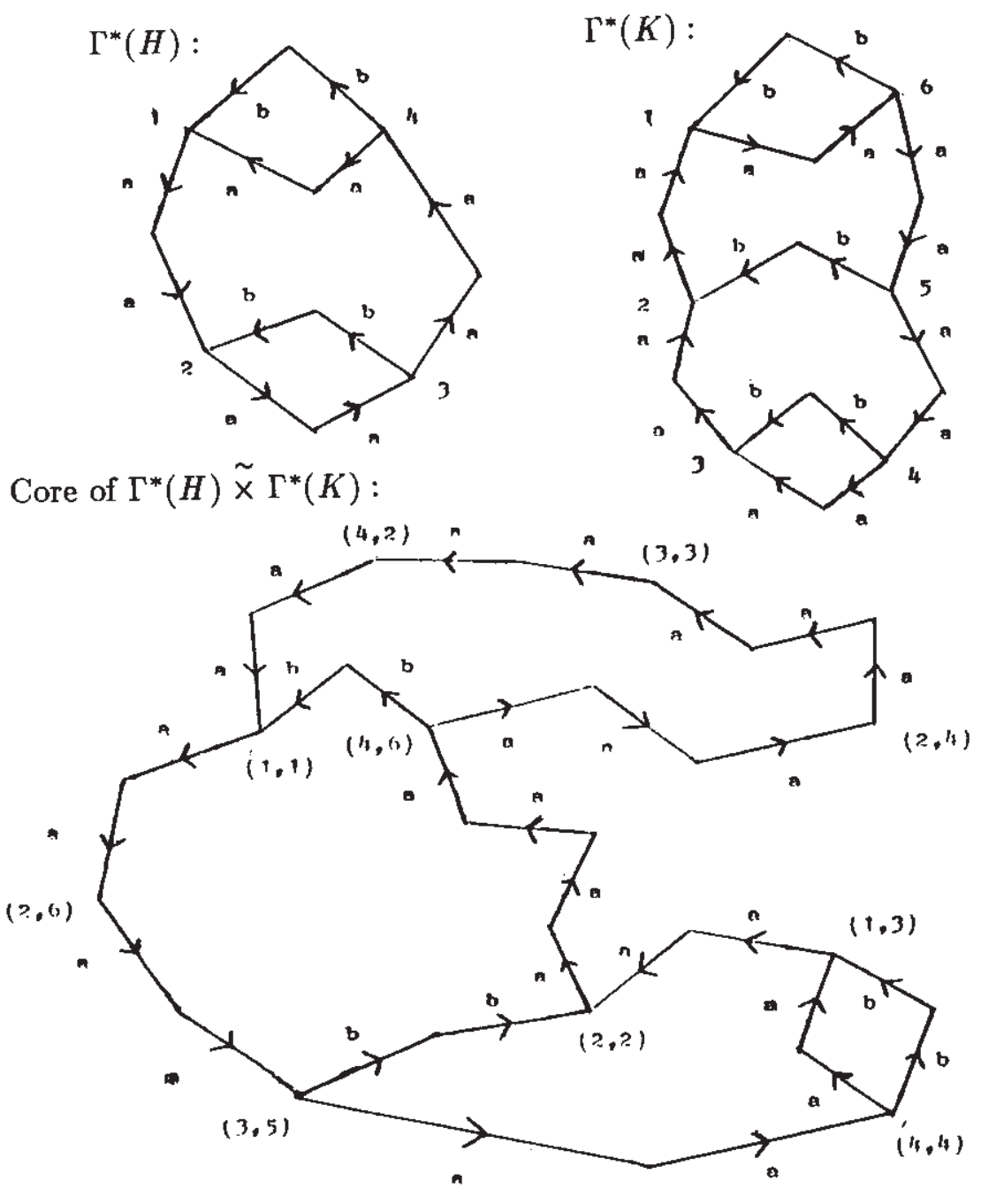
Example 3:

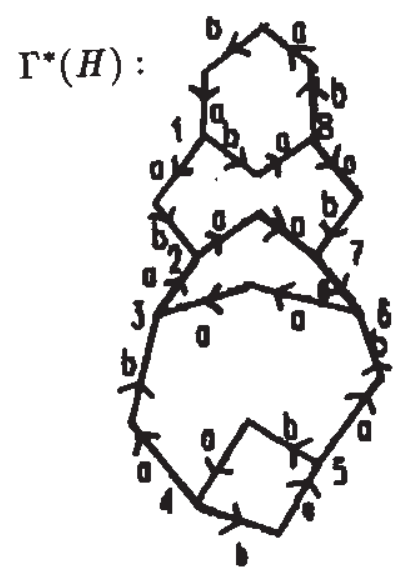

$\Gamma^{*}(K):$

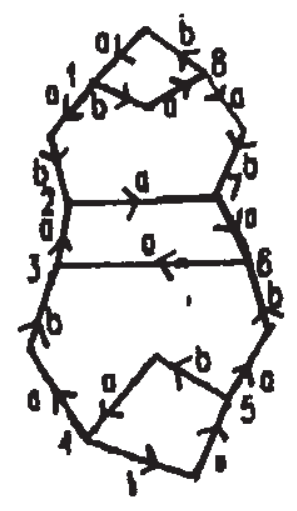

Core of $\Gamma^{*}(H) \tilde{x} \Gamma^{*}(K)$ :

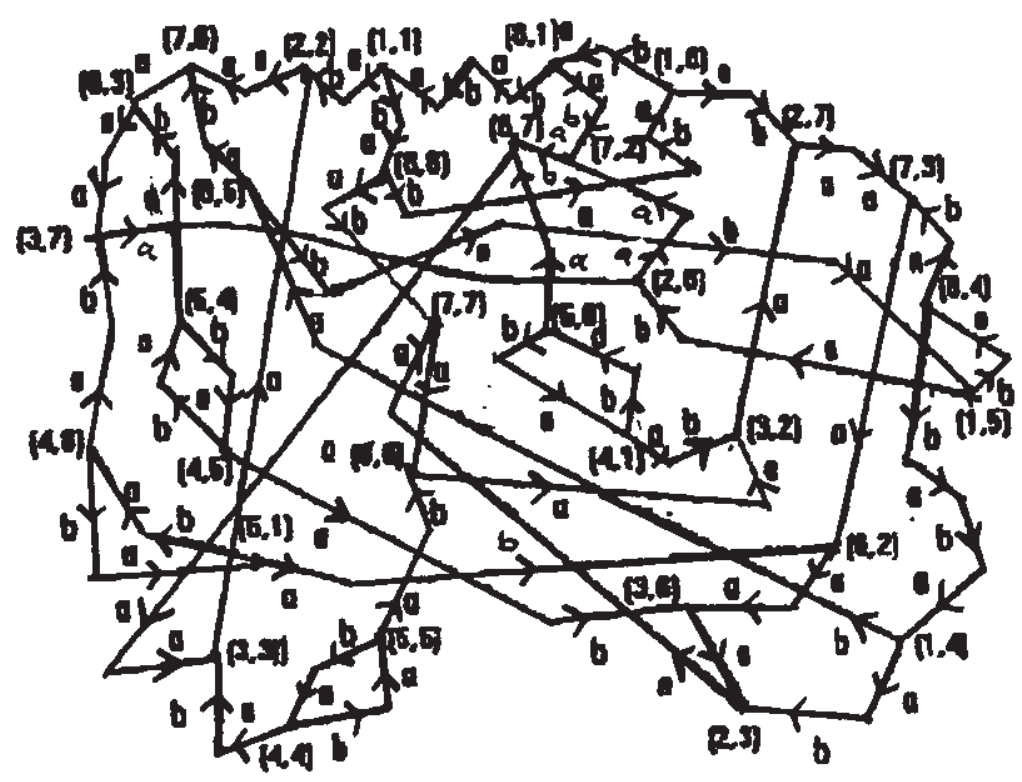




\section{References}

[1] Burns, R.G., A note on free group. Amer. Math. Soc. 23 (1969), 14-17.

[2] Burns, R.G., On the intersection of finitely generated subgroups of free groups. Math. Z. 119 (1971), 121-130.

[3] Gersten, S.M., Intersection of finitely generated subgroups of free groups and resolutions of graphs. Intrent. Math. 71 (1983), 567-591.

[4] Howson, A.G., On the intersection of finitely generated free groups. J. Lon. Math. Soc. 29 (1954), 428-434.

[5] Imrich, W., Subgroup theorem on groups. Combinatorial Math. V, 1-27 (lecture note in mathematices, 622. Springer-Verlag, Berlin, Heidelberg, New York, 1977).

[6] Imrich, W., On finitely generated subgroups of free groups. Arch. Math. 28 (1977), 21-24.

[7] Lyndon, R.C. and Schupp, P.E., Combinatorial group theory. Engebniss vol. 89, Berlin - Heidelberg - New York: Springer (1977).

[8] Neumann, H., On the intersection of finitely generated free groups, Math. Debrecen 4 (1955-56), 186-189.

[9] Neumann, H., On the intersection of finitely generated free groups: Addendum. Math Debrecen 5, (1957-50) 128.

[10] Nickolas, P., Intersection of finitely generated free groups. Bull. Austral. Math. Soc. Vol. 31 (1985), 339-349.

[11] Magnus, W., Karrass, A. and Solitaar, D., Combinatorial group theory. New York Wiley (1966).

[12] Sarvatius, B., A short proof of a theorem of Burns. Math. Z. 184 (1983), 133-137.

[13] Stallings, J.R., Topology of finite graphs. Invent. Math. 71 (1983), 551-565.

P.O. Box 4286

Ádhamiyah

BAGHDAD

IRAQ
Recibido: 6 de Junio, 1994

Revisado: 25 de Abril, 1995 